\title{
DEFINICIÓN Y ESTIMACIÓN DE TIPOS Y NIVELES DE USO TECNOLÓGICO: UNA APROXIMACIÓN A PARTIR DE ESTUDIANTES DE RECIÉN INGRESO A LA UNIVERSIDAD
}

\author{
Patricio Henríquez-Ritchie; sanbacayo@hotmail.com \\ Javier Organista-Sandoval; javor@uabc.mx \\ Universidad Autónoma de Baja California, México.
}

\section{RESUMEN.}

La investigación expuesta a continuación tuvo como objetivo principal definir y estimar los tipos y niveles de uso tecnológico en estudiantes que ingresan al nivel educativo superior y analizar su eventual relación con otras variables (personales, socioeconómicas, capacitación tecnológica previa, académicas). Se sitúa en la Facultad de Ciencias Administrativas y Sociales (FCAyS) de la Universidad Autónoma de Baja California (UABC), incluyendo a una parte de los estudiantes que ingresaron el primer semestre de 2008 (2008-1) en sus diferentes troncos comunes y licenciaturas. Los resultados evidencian que los principales tipos de tecnología computacional que los estudiantes utilizan más frecuente y hábilmente no coinciden necesariamente con aquellos programas/medios que consideran más importantes como apoyo en el marco de sus estudios. A su vez, los niveles de uso de la tecnología computacional se pueden definir a partir de la cantidad y calidad de uso del PC de los estudiantes y dichos niveles se muestran asociados a ciertas variables como género, edad, posesión de PC e Internet en el hogar y capacitación tecnológica previa.

PALABRAS CLAVES: uso tecnológico, tipos de uso tecnológico, niveles de uso tecnológico.

\section{ABSTRACT.}

The principal goal of the investigation exposed to continuation was to define and to measure the types and levels of technological use in students of recently entry to the university and analyzing the eventual relation with other variables (personal, socioeconomic, technological previous training, academic). Situate in the Faculty of Administrative and Social Sciences (FCAyS) of the Autonomous University of Baja California (UABC), including to a part of the students that entry in the first semester of the 2008 (2008-1) in his different licentiates. The results demonstrate that the principal types of technology that the students use more frequent and skillfully they do not coincide necessarily with those programs/means that they consider to be more important as support in the frame of their studies. In turn, the levels of technology use can be defined from the quantity and quality of use of the PC of the students and the above mentioned levels they prove to be partners to certain variables as genre, age, possession of PC and Internet in home and technological previous training.

KEY WORDS: technology use, types of technology use, levels of technology use. 


\section{INTRODUCCIÓN.}

El gran avance de las tecnologías de la información y comunicación (TIC) en la sociedad, desde mediados de la segunda mitad del siglo XX, ha propiciado el desarrollo de líneas de investigación abocadas a explorar el impacto de dichas tecnologías en el ámbito educativo.

En este sentido, ciertas investigaciones han apuntado al estudio del efecto que los medios tecnológicos tienen en los procesos educativos; es decir, las ventajas y desventajas que se presentan en comparación con el formato de clase tradicional ${ }^{1}$. Por su parte, Bebell, O’Dwyer, Russell y Hoffman (2007) aseveran que los estudios al respecto se pueden dividir en dos grandes grupos. Por un lado, aquellas investigaciones que intentan indagar de qué manera las dinámicas pedagógicas se ven impactadas con el uso de recursos tecnológicos y la evolución que sufren dichas dinámicas con su introducción. Y por otro lado, aquellos estudios que exploran los efectos de los medios tecnológicos como herramientas pedagógicas más que indagar el proceso fundamental de su incorporación.

No obstante, antes de investigar el impacto de las TIC en los procesos educativos y explorar cómo se ven trastocadas las dinámicas de enseñanza-aprendizaje con su incorporación, los estudios deben: i) establecer con claridad cómo están usando la tecnología tanto docentes como alumnos, y ii) construir formas de medición del uso tecnológico válidas y confiables (Bebell et al., 2007). Y precisamente en este punto es donde se ubica la presente investigación. Éste es el primer paso para entender de qué manera usan estos recursos los protagonistas de los procesos educativos y analizar cómo se relaciona dicho uso con otras variables personales, socioeconómicas y académicas, por mencionar algunas.

\section{REFERENTES TEÓRICOS.}

Uno de los principales usos de la tecnología se remite a la aplicación de las diversas funciones que derivan de la computadora y que está asociado tanto a labores académicas como extraacadémicas de los estudiantes. O’Dwyer, Russell y Bebell (2005) consideran el uso tecnológico como un concepto multidimensional; es decir, compuesto de múltiples variables que interactúan entre sí y que deben considerarse para su medición. Los mencionados autores proponen que dicho constructo se estime a partir de dos aproximaciones: la frecuencia de uso, que ayudaría a establecer un índice general de utilización, y las múltiples categorías tecnológicas utilizadas en las dinámicas educativas (Bebell, Russell y O’Dwyer, 2004).

Por un lado, los tipos de uso tecnológico se pueden representar mediante los múltiples programas/medios computacionales que comúnmente utilizan los protagonistas de los procesos educativos. Becker, Wong y Ravitz (1999) definen los tipos de uso tecnológico a partir de programas/medios tales como procesador de texto, hojas de cálculo/bases de datos, programas de presentación, WWW, correo electrónico, entre otros. Sin embargo, detrás de estos tipos de tecnología computacional se encuentra asociado el objetivo que cada usuario le otorga en el marco de sus necesidades académicas o extra-académicas. En el caso de los estudiantes, el uso de

\footnotetext{
${ }^{1}$ Chadwick (1997) caracteriza el modelo de enseñanza-aprendizaje tradicional según los siguientes elementos: el medio principal a través del cual se transmite la información está representado por el maestro; la forma de presentación es mayormente verbal $u$ oral; el rol de los estudiantes está relegado a receptores pasivos de información; la enseñanza se lleva a cabo generalmente en forma grupal; la responsabilidad principal del aprendizaje queda en manos del estudiante.
} 
tecnología se dirige principalmente a interactuar con los equipos y programas de cómputo ya sea con propósitos recreativos (programas de simulación y exploración, juegos y recreación, etc.), informativos (WWW, multimedia, entre otros) o académicos (procesadores de texto, programas de presentación, hojas de cálculo, etc.).

Por otro lado, los niveles de uso tecnológico se van a definir a partir de tres elementos principales: la frecuencia de uso, la profundidad en el conocimiento y aplicación de la tecnología computacional y la habilidad de uso por parte de los usuarios. La frecuencia de uso tecnológico refiere al número de ocasiones que un usuario utiliza la computadora durante un periodo de tiempo determinado.

En cuanto a la profundidad o inmersión de los usuarios frente a los recursos computacionales se han elaborado distintas escalas para su estimación. De especial interés para este estudio es la escala de adopción de la tecnología (Stages of Adoption of Technology) aplicada a docentes de ocho estados de México hacia 1998 (Morales, 1999). Ésta consta de seis etapas jerárquicas a partir de las cuales se estima la profundidad en el manejo, confianza y conocimiento de los recursos computacionales por parte de los usuarios. Para determinar el nivel o profundidad de manejo de los recursos del Web, Lowther, Jones y Plants (2000) establecieron una escala de inmersión análoga con cinco niveles jerárquicos de uso. Finalmente, la habilidad de uso computación refiere a la utilización efectiva y productiva de los recursos computacionales.

En resumen, los tipos de uso tecnológico se definirán a partir de:

- Tipos de programas: procesador de texto, hojas de cálculo/bases de datos, programas de presentación (por ejemplo, Word, Excel y PowerPoint respectivamente), Internet, multimedia, CD-ROM, entre otros.

- Finalidades de uso: objetivos que cada usuario le asigna a la aplicación del recurso computacional.

Para los niveles de uso tecnológico se considerarán las siguientes dimensiones:

- Frecuencia de uso: recurrencia en el uso de la computadora.

- Inmersión o profundidad de uso: capacidad de realización y confianza en la relación usuario-computadora.

- Habilidad de uso: utilización efectiva, eficaz y productiva de las funciones computacionales.

De esta manera, para estimar los tipos y niveles de uso tecnológico se debe recurrir a un instrumento que integre las diversas dimensiones que se encuentran detrás del uso computacional, tanto en la esfera académica como extra-académica de los usuarios. La convergencia de estas dimensiones puede transformarse en la clave para entender de mejor manera las formas en que se utiliza la tecnología computacional y los niveles de uso de la misma. El primer paso es desarrollar un instrumento de medición que permita observar qué tipos de programas computacionales son los más recurrentemente utilizados por los estudiantes, los objetivos de uso de las computadoras y el nivel de profundidad de uso del recurso computacional. 


\section{OBJETIVOS.}

- Definir y estimar los tipos y niveles de uso tecnológico en estudiantes de recién ingreso (período 2008-1) a la Facultad de Ciencias Administrativas y Sociales (FCAyS) de la Universidad Autónoma de Baja California (UABC).

- Analizar la eventual relación del nivel de uso tecnológico con variables personales, socioeconómicas, académicas y de capacitación tecnológica previa.

\section{MÉTODO.}

\subsection{Contexto espacio-temporal y participantes}

Se consideraron cinco troncos comunes/licenciaturas de la FCAyS de la UABC, campus Ensenada: i) área de administración (TCadm), ii) área de ciencias sociales (TCSOc), iii) Informatica, iv) Derecho y v) semi-escolarizado (TCsemiesc). En este último, los estudiantes asisten a clases sólo los fines de semana. El estudio se realizó durante el primer semestre de 2008. El número de participantes en la investigación y su porcentaje con relación al total de inscritos se muestran en la tabla 1.

\begin{tabular}{|l|l|l|l|l|l|l|l|}
\hline \multicolumn{2}{|c|}{} & TCad & TCsoc & Informáti & Derecho & TCsemie & GLOBAL \\
\hline \multicolumn{2}{|l|}{ Total inscritos } & 453 & 431 & 99 & 418 & 97 & $\mathbf{1 4 9 8}$ \\
\hline \multirow{2}{*}{$\begin{array}{l}\text { Participant } \\
\text { es }\end{array}$} & $\boldsymbol{n}$ & 100 & 144 & 27 & 146 & 21 & $\mathbf{4 3 8}$ \\
\cline { 2 - 8 } & $\%$ & 22.1 & 33.4 & 27.7 & 34.9 & 21.7 & $\mathbf{2 9 . 2}$ \\
\hline
\end{tabular}

Tabla 1. Alumnos inscritos en la FCAyS (período 2008-1) y participantes en la investigación.

\subsection{Instrumentos}

Se elaboró un instrumento llamado Encuesta de uso tecnológico para estudiantes, sobre la base de la revisión bibliográfica relacionada (anexo 1). Las dimensiones que componen dicho instrumento y sus principales variables se presentan a continuación.

- Datos personales. Relacionada con la identificación de los participantes: nombre, edad, género, tronco común/licenciatura.

- Trayectoria escolar y capacitación tecnológica previa. Comprende el historial académico de los participantes, principalmente la media de calificaciones de bachillerato. A su vez, se consideraron los años de experiencia de uso del PC y los antecedentes de capacitación en el área computacional, como factores significativos para indagar los tipos de uso de la computadora dentro de contextos educativos (Van Braak, Tondeur y Valke, 2004; Morales, 1999)

- Datos socioeconómicos y disponibilidad de PC e Internet en el hogar. Se relaciona con las condiciones socioeconómicas de los participantes, principalmente la escolaridad del padre y madre y la posesión de PC/Internet en el hogar (Organista y Backhoff, 1999; O'Dwyer et al., 2005). 
- Tipos y niveles de uso tecnológico. A partir de la perspectiva trazada por Bebell et al. (2004); Russell, O’Dwyer, Bebell y Miranda (2004) y O'Dwyer et al. (2005), el uso tecnológico es visto como un constructo multivariado donde se integran tanto los tipos como los niveles de uso, en desmedro de aquellas escalas de medición que buscan estimar cada uno de estos constructos de manera independiente. Se agregaron reactivos para indagar la frecuencia de uso (escala Likert de cinco puntos entre nunca y los 7 días de la semana) y la habilidad de uso (escala Likert de 5 puntos entre pésimo y excelente) de los participantes por programa. Se identificaron 12 tipos de programas/medios computacionales a partir de la revisión bibliográfica. También se incluyó una escala de 11 actividades relacionadas con el uso de la computadora para estimar la capacidad de realización de los participantes (Likert de 5 pasos entre pésimo y excelente), componente importante a considerar dentro del uso tecnológico (Directorio para la Educación y Cultura, 2003). A su vez, a partir de una lista de 9 objetivos de uso, los participantes eligieron los 3 más importantes según la importancia que le asignan en sus estudios. Para estimar el nivel de inmersión en el uso tecnológico, se consideró la escala de nivel de inmersión en el manejo/uso de la computadora (Morales, 1999) y otra relacionada con la inmersión del usuario en la Web (Lowther, Jones y Plants, 2000).

- Opinión acerca de la utilidad de la tecnología en sus estudios. En esta dimensión se buscó conocer la opinión de los participantes en torno al beneficio de utilizar a la computadora como apoyo a las labores académicas (Marcinkiewicz, 1993; Marcinkiewicz 1994; Christensen, 1998; Knezek y Christensen 1995; Russell et al., 2004). Para ello, se incluyeron un ítem dicotómico para que los participantes ubiquen su opinión acerca del impacto de la tecnología en el aprendizaje y 13 enunciados relacionados con el rol de la computadora en sus actividades académicas y a nivel personal, las cuales debieron ser calificadas según una escala Likert de cinco puntos entre el acuerdo y el desacuerdo total.

Un resumen de las dimensiones y variables consideradas en la encuesta se muestra en la tabla 2.

\begin{tabular}{|l|ll|}
\hline Dimensiones & \multicolumn{2}{l|}{ Variables } \\
\hline & 1.1 & Nombre \\
& 1.2 & Carrera/tronco común que cursa \\
1. Datos personales & 1.3 & Edad \\
& 1.4 & Género \\
& 1.5 & Estado civil \\
& 1.6 & Número de dependientes \\
\hline \multirow{2}{*}{ 2.Trayectoria escolar } & 2.1 & Promedio bachillerato \\
capacitación tecnológica & 2.2 & Tipo de escuela de procedencia \\
& 2.3 & Años de experiencia en el uso del PC \\
& 2.4 & Capacitación tecnológica previa \\
3. Datos socioeconómicos y y & 3.1 & Casa propia/rentada \\
disponibilidad de PC e & 3.3 & Púmero de personas en casa \\
internet & 3.4 & Lugar de acceso a los servicios de internet \\
& 3.5 & Escolaridad padre/madre \\
& 3.6 & Ocupación padre/madre \\
\hline
\end{tabular}




\begin{tabular}{|l|ll|}
\hline & 4.1 & Tipo de equipo personal \\
& 4.2 & Horas diarias aproximadas de uso del PC \\
4.3 & Frecuencia de uso por programa/medio \\
4.Tipo y nivel de uso & 4.4 & Tres programas más importantes \\
tecnológico & 4.5 & Habilidad de uso por programa/medio \\
& 4.6 & Capacidad de realización por actividad \\
& 4.7 & Tres principales objetivos de uso del PC \\
& 4.8 & Nivel de inmersión en el uso del PC \\
& 4.9 & Nivel inmersión en el uso del web \\
\hline 5.Opinión acerca de la utilidad & 5.1 & Percepción de la relevancia de la computadora en \\
de la tecnología & el aprendizaje \\
& 5.2 & Utilidad/rol de la computadora \\
\hline
\end{tabular}

Tabla 2. Dimensiones y variables de la encuesta de uso tecnológico para estudiantes.

Otro instrumento utilizado en la investigación fue el listado de media de calificaciones de los participantes al término del primer semestre de estudios (2008-1).

\subsection{Procedimiento}

El procedimiento consistió de 2 etapas. En la primera, se aplicó la encuesta al inicio del ciclo escolar, específicamente durante la realización del curso de inducción que la universidad ofrece a sus nuevos estudiantes. Una segunda etapa consistió en solicitar a la dependencia administrativa de la universidad su apoyo para la obtención de las medias de calificaciones al final del semestre para aquellos estudiantes que respondieron la encuesta.

\subsection{Análisis de datos}

Se utilizó el programa estadístico SPSS $^{\oplus}$ para el procesamiento de los datos. Se calcularon indicadores descriptivos de tendencia central, de dispersión y se elaboraron tablas de frecuencias. Se aplicaron técnicas de análisis de clasificación (conglomerados k-medias). 


\section{RESULTADOS.}

\subsection{Características generales de los participantes.}

El $66 \%$ del total de participantes fueron mujeres. Esta tendencia se mantuvo en todos los troncos comunes con excepción de Informática, donde los hombres representaron más de la mitad de los participantes (55.6\%). Por su parte, la edad media de los participantes fue de 20.6 años. En cuanto a la posesión de PC e Internet en el hogar, más de la mitad (53.6\%) disponen de dicho equipo/servicio (ver tabla 5.1).

\begin{tabular}{|c|c|c|c|c|c|c|c|c|c|c|c|c|c|}
\hline & \multicolumn{2}{|c|}{ TCadm } & \multicolumn{2}{|c|}{ TCsoc } & \multicolumn{2}{|c|}{ Informática } & \multicolumn{2}{|c|}{ Derecho } & \multicolumn{2}{|c|}{ TCsemiesc } & \multicolumn{2}{|c|}{ Global } \\
\hline & & $n$ & $\%$ & $n$ & $\%$ & $n$ & $\%$ & $n$ & $\%$ & $n$ & $\%$ & $n$ & $\%$ \\
\hline \multirow{2}{*}{ Género } & Hombres & 37 & 37.0 & 35 & 24.3 & 15 & 55.6 & 56 & 38.4 & 4 & 19.0 & 147 & 33.6 \\
\hline & Mujeres & 63 & 63.0 & 109 & 75.7 & 12 & 44.4 & 90 & 61.6 & 17 & 81.0 & 291 & 66.4 \\
\hline \multicolumn{2}{|l|}{$\operatorname{Sin} P C$} & 26 & 26.0 & 31 & 21.7 & 5 & 18.5 & 29 & 20.1 & 4 & 19.0 & 5 & 21.8 \\
\hline \multicolumn{2}{|l|}{ Con PC } & 27 & 27.0 & 29 & 20.3 & 8 & 29.6 & 37 & 25.7 & 6 & 28.6 & 107 & 24.6 \\
\hline \multicolumn{2}{|c|}{ PC+Internet } & 47 & 47.0 & 83 & 58.0 & 14 & 51.9 & 78 & 54.2 & 11 & 52.4 & 233 & 53.6 \\
\hline
\end{tabular}

En la figura 5.1 se observa el grado de capacitación en el área computacional de los participantes. Aproximadamente el $60 \%$ de los alumnos manifestó tener un grado de capacitación de intermedio o avanzado. Esto implica el manejo de ciertos programas tales como procesadores de texto, programas para crear presentaciones y hojas de cálculo/bases de datos, entre otros. Llama la atención que de manera general, un porcentaje cercano al $15 \%$ no tenga ninguna capacitación previa.

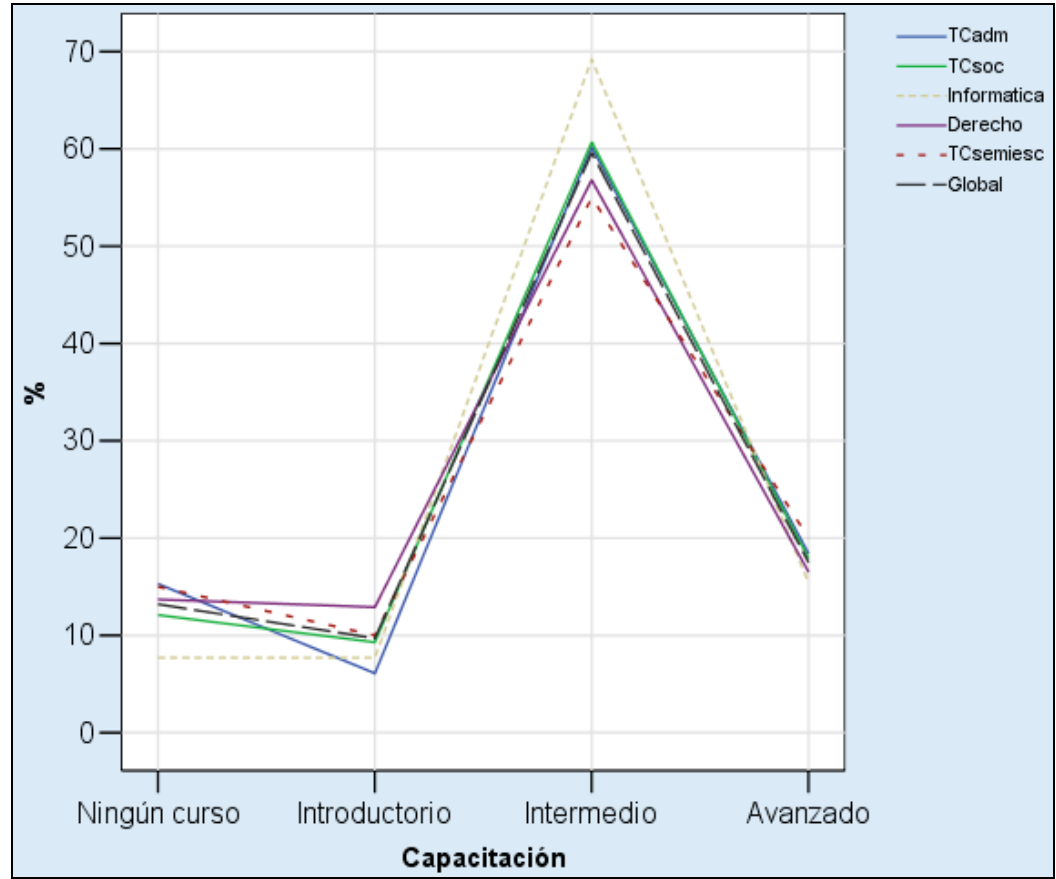

Figura 5.1. Grado de capacitación en el área computacional de los participantes 


\subsection{Tipos y niveles de uso tecnológico.}

Para estimar el nivel de profundidad en el manejo/uso de la computadora se consideraron 6 niveles jerárquicos de inmersión, de acuerdo con la escala de Morales (1999), mismos que a continuación se describen. En el i) nivel de conciencia, se conoce la existencia de la tecnología, pero se evita su uso ya que genera angustia y ansiedad; ii) aprendiendo el proceso, se incursiona en el aprendizaje básico en manejo de la computadora; iii) entendimiento y aplicación, se comprende la utilidad de la tecnología; iv) familiaridad y confianza, se adquiere confianza y se percibe normal el uso de la computadora; $v$ ) adaptación a otros contextos, la computadora es vista como una herramienta útil en contextos diversos y no hay temores antes su uso y vi) aplicación creativa, se disponen y utilizan programas diversos en labores académicas y extra-académicas.

A nivel global, cerca del $75 \%$ de de los participantes se agrupan en los niveles altos (aplicación creativa y adaptación a otros contextos), lo cual sugiere un buen nivel de manejo computacional de los participantes en el estudio. Llama la atención el bajo porcentaje (14\%) de participantes que se ubicó en los tres niveles más básicos de manejo/uso de la tecnología computacional a nivel general (ver fig. 5.2).

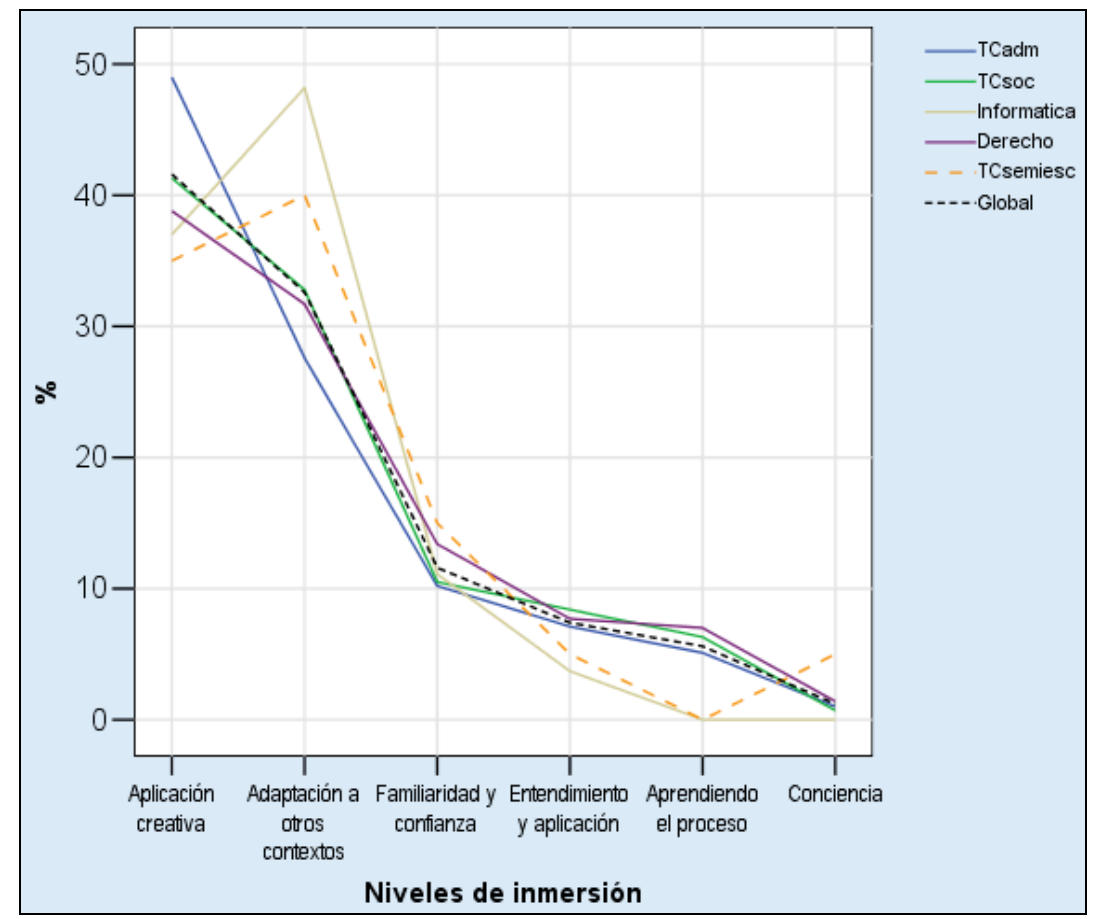

Figura 5.2. Nivel de inmersión tecnológica para cada tronco común

Para el nivel de inmersión de uso de la Web se consideraron 5 etapas jerárquicas, descritas a continuación. En el nivel inferior o básico llamado i) informativo, solo se consulta información general, p. ej. noticias, avisos. En el nivel ii) suplementario, se recupera información adicional para tareas o trabajos; en el nivel iii) esencial, la Web es necesaria para ser productivo y eficiente en los estudios; en el nivel iv) comunitario, se accede frecuentemente a Internet y se aporta información en algún sitio web. Finalmente, el nivel v) inmersivo, se depende de la Web para la búsqueda de información, procesamiento y comunicación. 
A nivel general, el $12-13 \%$ de los participantes refieren tener un buen nivel de manejo de la Web en los niveles inmersivo o comunitario respectivamente. Los mayores porcentajes (30-35\%) ocurren en los niveles esencial o suplementario (ver figura 5.3).

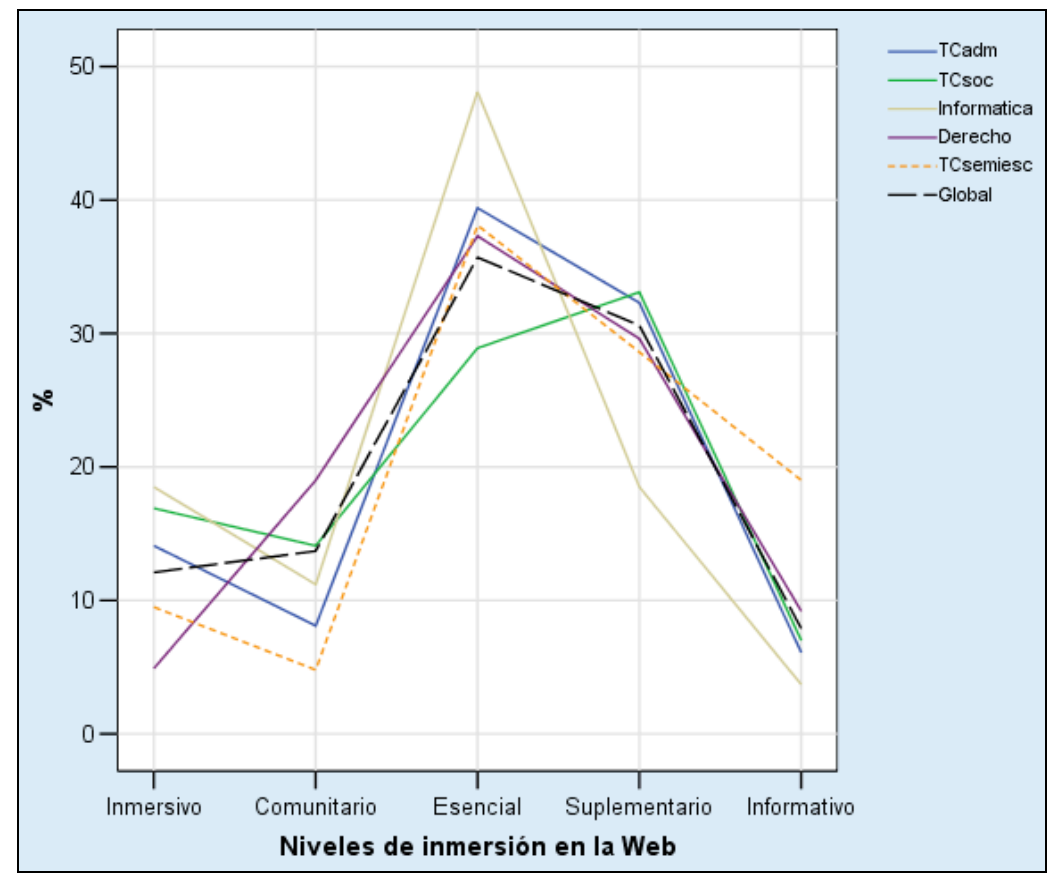

Figura 5.3. Nivel de inmersión en la Web, según el tronco común.

Con relación a la capacidad de realización de ciertas actividades relacionadas con el uso de la computadora, se estimó a partir de una escala de Likert de 5 pasos entre pésimo y excelente para un total de 11 actividades. Las tres principales actividades realizadas con la computadora fueron: i) búsqueda de información en Internet; ii) comunicación vía electrónica y iii) transferencia de archivos entre medios (carpetas, CD, USB, entre otros). Por su parte, las actividades menos realizadas, fueron: i) manejo de hojas de cálculo; ii) procesamiento estadístico de datos y iii) manejo de bases de datos (ver tabla 5.2).

\begin{tabular}{|c|c|c|c|c|c|c|c|c|c|c|c|c|}
\hline \multirow{2}{*}{$\begin{array}{l}\text { ACTIVIDADE } \\
S\end{array}$} & \multicolumn{2}{|c|}{ TCadm } & \multicolumn{2}{|c|}{ TCsoc } & \multicolumn{2}{|c|}{ Informátic } & \multicolumn{2}{|c|}{ Derecho } & \multicolumn{2}{|c|}{ TCsemiesc } & \multicolumn{2}{|c|}{ Global } \\
\hline & $\begin{array}{l}\text { Med } \\
\text { ia }\end{array}$ & D.E. & $\begin{array}{l}\text { Me } \\
\text { dia }\end{array}$ & D.E. & $\begin{array}{l}\text { Me } \\
\text { dia }\end{array}$ & D.E. & $\begin{array}{l}\text { Med } \\
\text { ia }\end{array}$ & D.E. & $\begin{array}{l}\text { Me } \\
\text { dia }\end{array}$ & D.E. & $\begin{array}{l}\text { Medi } \\
a\end{array}$ & D.E. \\
\hline Internet & 3.2 & 0.7 & 3.2 & 0.8 & 3.2 & 0.9 & 3.1 & 0.9 & 2.8 & 1.0 & 3.1 & 0.9 \\
\hline $\begin{array}{l}\text { Comunicaci } \\
\text { ón }\end{array}$ & 2.8 & 1.1 & 3.0 & 1.0 & 2.8 & 1.2 & 2.9 & 1.1 & 2.8 & 1.2 & 2.9 & 1.1 \\
\hline $\begin{array}{l}\text { Transferenc } \\
\text { ia }\end{array}$ & 2.9 & 1.0 & 2.9 & 1.0 & 3.4 & 0.8 & 2.8 & 1.1 & 2.4 & 1.1 & 2.9 & 1.0 \\
\hline $\begin{array}{l}\text { Hojas de } \\
\text { cálc. }\end{array}$ & 1.7 & 1.0 & 1.4 & 1.0 & 1.5 & 0.8 & 1.6 & 1.0 & 1.2 & 1.0 & 1.6 & 1.0 \\
\hline Proc.Estad & 1.2 & 0.8 & 1.2 & 0.9 & 1.2 & 1.1 & 1.3 & 1.0 & 0.6 & 0.7 & 1.2 & 0.9 \\
\hline $\begin{array}{l}\text { Base de } \\
\text { datos }\end{array}$ & 1.1 & 0.9 & 0.9 & 0.8 & 1.2 & 0.8 & 1.1 & 0.9 & 0.8 & 0.9 & 1.0 & 0.9 \\
\hline
\end{tabular}

Tabla 5.2. Descriptivos básicos para la variable capacidad de realización de actividades relacionadas la computadora. 
En coincidencia con lo anterior, como se observa en la tabla 5.3 los principales programas o medios mayormente utilizados fueron: correo electrónico, WWW, juegos/recreación y procesador de texto, los cuales tuvieron como objetivo, la realización de trabajos académicos como proyectos, ensayos, tareas, etc., búsqueda de información y/o comunicación entre estudiantes. Por su parte, los programas menos utilizados fueron los paquetes matemáticos, estadísticos y de diseño.

\begin{tabular}{|l|l|l|c|c|}
\hline \multicolumn{2}{|l|}{ FRECUENCIA } & \multirow{2}{*}{ PROGRAMAS/MEDIOS } & \multicolumn{2}{l|}{ HABILIDAD $^{*}$} \\
\cline { 4 - 5 } Medi & Lugar & & Lugar & Media \\
\hline 3.3 & 1 & Correo electrónico & 1 & 3.2 \\
\hline 3.2 & 2 & WWW & 2 & 3.0 \\
\hline 2.7 & 3 & Juegos/recreación & 3 & 2.8 \\
\hline 2.4 & 4 & Procesador de texto & 7 & 2.9 \\
\hline 2.2 & 5 & Programas para manejo multimedia & 6 & 2.3 \\
\hline 2.0 & 6 & Foros asincrónicos y chats & 5 & 2.4 \\
\hline 1.7 & 7 & Programas de presentación & 7 & 2.3 \\
\hline 1.5 & 8 & Enciclopedias/referencias en CD-ROM & 9 & 1.8 \\
\hline 1.3 & 9 & Blogs & 8 & 2.0 \\
\hline 1.3 & 9 & Hojas de cálculo/bases de datos & 10 & 1.4 \\
\hline 0.8 & 10 & Programas gráficos & 11 & 1.1 \\
\hline 0.5 & 11 & Paquetes matemáticos, estadísticos $\boldsymbol{y}$ de & 11 \\
\hline \multirow{2}{*}{$\begin{array}{l}\text { * La estimación de la media estadística se hizo considerando la codificación (0-4) de la escala de Likert } \\
\text { utilizada para estimar tanto la frecuencia como la habilidad de uso de la computadora. }\end{array}$} \\
\hline
\end{tabular}

Tabla 5.3. Principales tipos de uso tecnológico según frecuencia y habilidad de uso de programas/medios.

Con el propósito de obtener una clasificación asociada a los niveles de uso tecnológico de los participantes se llevó a cabo un análisis de conglomerados (K-Means Clusters) a partir de las variables: nivel de inmersión en el uso de la tecnología computacional, nivel de inmersión en la Web, frecuencia de uso, habilidad de uso y capacidad de realización de tareas. Los resultados del análisis ANOVA, muestran que las principales aportaciones a la clasificación se deben a las variables: nivel de inmersión en el uso de la tecnología computacional y nivel de inmersión en la Web. La tabla 5.4 muestra los centroides para cada uno de los tres conglomerados.

\begin{tabular}{|c|c|c|c|}
\hline \multirow[b]{2}{*}{ MEDIAS } & \multicolumn{3}{|c|}{ CONGLOMERADOS } \\
\hline & $1(n=90)$ & $\begin{array}{l}2 \\
(n=205)\end{array}$ & $3=(99)$ \\
\hline $\begin{array}{l}\text { Nivel de inmersión en el uso de tec. } \\
\text { computacional }^{1}\end{array}$ & 3 & 5 & 6 \\
\hline Nivel de inmersión en la Web $^{2}$ & 2 & 2 & 4 \\
\hline Frecuencia de uso & 1.3 & 2.0 & 2.3 \\
\hline Habilidad de uso & 1.6 & 2.5 & 2.7 \\
\hline Capacidad de realización de trabajos & 1.5 & 2.5 & 2.7 \\
\hline
\end{tabular}

Tabla 5.4. Valores medios para cada conglomerado según las variables de uso tecnológico 


\section{DISCUSIÓN}

Un resultado que llama la atención fue el alto grado de profundidad en el manejo/uso de la tecnología computacional de los participantes con base en la ubicación que arrojo la escala de adopción de la tecnología. A nivel global el 74\% de los estudiantes se ubicaron en las dos etapas más altas de dicha escala, lo que implica utilizar la computadora en variadas actividades sin temor ni ansiedad. En el caso de la escala de inmersión en la Web, el $66 \%$ de los estudiantes mostraron un nivel de profundidad moderado en su uso. Estos resultados son un reflejo de como la generación de recién ingreso a la universidad está constituida mayormente por jóvenes de los llamados 'nativos digitales' quienes desde su infancia han estado en contacto con dispositivos tecnológicos diversos.

De acuerdo con Morales (1999), quienes tienen una mayor frecuencia de uso computacional, una mejor experiencia previa y un grado de capacitación avanzado se asocian a una ubicación más alta en las etapas de adopción de la tecnología. Estos señalamientos coinciden con los hallazgos de esta investigación, ya que fueron precisamente quienes mostraron mayor frecuencia, habilidad y capacidad de realización de ciertas actividades relacionadas con el uso del PC los que se ubicaron significativamente en el nivel más alto de dicha escala.

La clasificación de tipos y niveles de uso tecnológico propuesta en los resultados es sugerente acerca de la forma en que los estudiantes usan los recursos computacionales y la profundidad en que se ubican con respecto a dicho manejo. En cuanto a los tipos de uso, queda en evidencia que hay tres programas/medios computacionales usados más frecuente y hábilmente por los estudiantes, a saber, correo electrónico, WWW, juegos y recreación. Esta tendencia coincide con los resultados de un estudio con estudiantes de secundaria del estado de Massachusetts, EE.UU. (Russell et al., 2004), donde las principales preferencias las obtuvieron Internet, correo electrónico, chat, juegos y diversión. Más allá de las diferencias contextuales entre las muestras de ambos estudios, lo cual impediría trazar comparaciones válidas, lo destacable son las tendencias hacia el uso de tipos de tecnología computacional similares entre jóvenes que se encuentran en etapas generacionales análogas.

No obstante lo anterior, cuando los alumnos del presente estudio seleccionaron los programas/medios que consideran más importantes como apoyo en sus estudios aparecen el procesador de texto y los programas de presentación en los dos primeros lugares. A su vez, cuando elijen los principales objetivos de uso de la computadora aparece en primer lugar elaborar proyectos, ensayos, tareas, etc. (seguido de buscar información y comunicarse por correo electrónico). De lo anterior se infiere que si bien en la práctica los estudiantes usan de manera más frecuente y hábil ciertos programas/medios, cuando se les pregunta por la importancia en su proceso académico apuntan a otros. Por ende, el primer objetivo de uso no se relaciona mayormente con los tres programas/medios usados con más frecuencia y mayor habilidad. De este modo, se vislumbra una dimensión práctica del uso tecnológico en la que los estudiantes usan más y manejan mejor ciertos programas/medios, frente a otra dimensión en la que el discurso de los participantes le asigna mayor trascendencia a otros programas/medios computacionales.

Así, de este modo, aquellos participantes que se ubicaron en los niveles más altos de uso de la tecnología computacional utilizan de manera más frecuente ciertos programas/medios relacionados con actividades extra-académicas, como por ejemplo aquellos relacionados juegos y recreación. A su vez, a partir de la habilidad aquellos estudiantes ubicados en el nivel más alto de uso de la computadora también dicen utilizar de mejor modo programas/medios recreacionales. 
Sin embargo, los objetivos de uso que le asignan a dicha tecnología están relacionados principalmente con su rol de educando.

La investigación aquí presentada alerta sobre de la importancia de conocer el nivel de habilidades tecnológicas que tienen los estudiantes al ingresar a la universidad ya que, en gran medida, la tecnología computacional y del web es una herramienta pedagógica que bien dirigida puede contribuir a su éxito académico. Sin embargo, no se debe olvidar que los maestros son actores importantes para motivar y dirigir esta herramienta, ya que son ellos quienes pueden promover o inhibir la utilización de las nuevas tecnologías.

\section{REFERENCIAS}

Bebell, D., Russell, M. y O'Dwyer, L. (2004). Measuring teachers' technology uses: Why multiplemeasures are more revealing. Journal of Research on Technology in Education, 37 (1), 45-63.

Bebell, D., O'Dwyer, L., Russell, M. y Hoffman, T. (2007). Advancing data collection in the digital age: methodological challenges and solutions in educational technology research. Boston, MA: Boston College, Technology and Assessment Study Collaborative. Paper Presented at the: Annual Meeting of American Educational Research Association Meeting, Chicago, IL. Consultado el 06 de junio de 2007,

http://www.bc.edu/research/intasc/aera/Methodological\%20challenges v2.2.pdf

Becker, H., Wong, Y. y Ravitz, J. (1999). Computer Use and Pedagogy in Co-NECT Schools, a comparative study. University of California, Irvine. Consultado el 6 de junio de 2007, en: http://crito.uci.edu/papers.asp?offset=160.

Chadwick, C. (1997). Tecnología educacional para el docente. Paidós: Barcelona.

Christensen, R. (1998). Effect of technology integration education on the attitudes of teachers and their students. Recuperado el 18 de febrero de 2007, en: http://www.tcet.unt.edu/research/dissert/rhondac/index.htm.

Directorio para la educación y cultura (2003). SESIUSS Project. Final Report. Programa SócratesMinerva, Comisión Europea. Consultado el 15 de octubre de 2007, en: http://www.intermedia.uib.no/seusiss/results.html

Knezek, G. A. y Christensen, R. (1995). A comparison of two computer curricular programs at a Texas Junior High School using the computer attitude questionnaire. Recuperado el 15 de febrero de 2007, en: http://www.tcet.unt.edu/research/techrept

Lowther, D., Jones, M. y Plants, R. (2000). Preparing tomorrow's teachers to use web-based education. En: Abbey, B. Instructional and cognitive impacts of web-based education. Hershey, PA: Idea Group Publishing.

Marcinkiewicz, H. (1993). Computers and teachers: factors influencing computer use in the classroom. Journal of Research on Computing in Education, 26 (2), 220-37. 
Marcinkiewicz, H. (1994). Subjective Norms Predicting Computer Use. Proceedings of Selected Research and Development Presentations at the 1994 National Convention of the Association for Educational Communications and Technology Sponsored by the Research and Theory Division, Nashville, TN. ERIC: ED373739.

Morales, C. (1999). Etapas de adopción de la tecnología informática al salón de clases. XV Simposio Internacional de Computación en la educación. Universidad de Guadalajara. SOMECE. Consultado el 01 de agosto de 2007, en: http://www.somece.org.mx/memorias/1999/inditema.htm

O’Dwyer, L., Russell, M. y Bebell, D. (2005). Identifying teacher, school, and district characteristics associated with middle and high school teachers' use of technology: a multilevel perspective. Journal of educational computing research, 33 (4), 369-393.

Organista, J. y Backhoff, E. (octubre-diciembre 1999). El uso de Internet para administrar tareas, exámenes y asesorías en la educación superior. Revista de la Educación Superior, 28 (112). Recuperado el 11 de mayo de 2007 en: http://www.anuies.mx/servicios/p anuies/index2.php?clave=publicaciones/revsup.

Russell, M., O'Dwyer, L., Bebell, D. y Miranda, H. (2004). Technical report for the USEIT study. Boston, MA: Boston College, Technology and Assessment Study Collaborative. Consultado el 06 de junio de 2007, en: http://escholarship.bc.edu/cgi/viewcontent.cgi?article=1024\&context=intasc

Van Braak, J., Tondeur, J. y Valke, M. (2004). Explaining different types of computer use among primary school teachers. European Journal of Psychology of Education, 19 (4), 407-422.

\section{Para citar este artículo:}

HENRÍQUEZ, Patricio; ORGANISTA, Javier, (2009) "Definición y estimación de tipos y niveles de uso tecnológico: una aproximación a partir de estudiantes de recién ingreso a la universidad» [artículo en línea]. EDUTEC, Revista Electrónica de Tecnología Educativa. Núm. 30 / Noviembre 2009. [Fecha de consulta: $\mathrm{dd} / \mathrm{mm} / \mathrm{aa}]$.

http://edutec.rediris.es/revelec2/revelec30/

ISSN 1135-9250. 


\section{Anexo 1}

\section{Encuesta de uso tecnológico para estudiantes}

Como parte de un trabajo de investigación del programa de Maestría del Instituto de Investigación y Desarrollo Educativo (IIDE) de la UABC, se ha desarrollado esta encuesta para recopilar información acerca de los tipos y niveles de uso de la computadora en estudiantes de la Facultad de Ciencias Administrativas y Sociales (FCAyS). Su objetivo es analizar las relaciones entre el uso de la computadora y variables de desempeño académico en los alumnos recién ingresados al nivel educativo superior. La información aquí manejada es absolutamente confidencial. Por favor, ayúdanos con nuestra investigación contestando la encuesta de forma honesta. De antemano, gracias por tu colaboración.

\section{INSTRUCCIONES:}

A continuación, encontrarás una serie de preguntas. Por favor, lee atentamente y contesta la información solicitada en cada recuadro. Para el caso de las preguntas con más de una opción, marca con una X el cuadrado que mejor representa tu alternativa.

\section{DATOS PERSONALES}

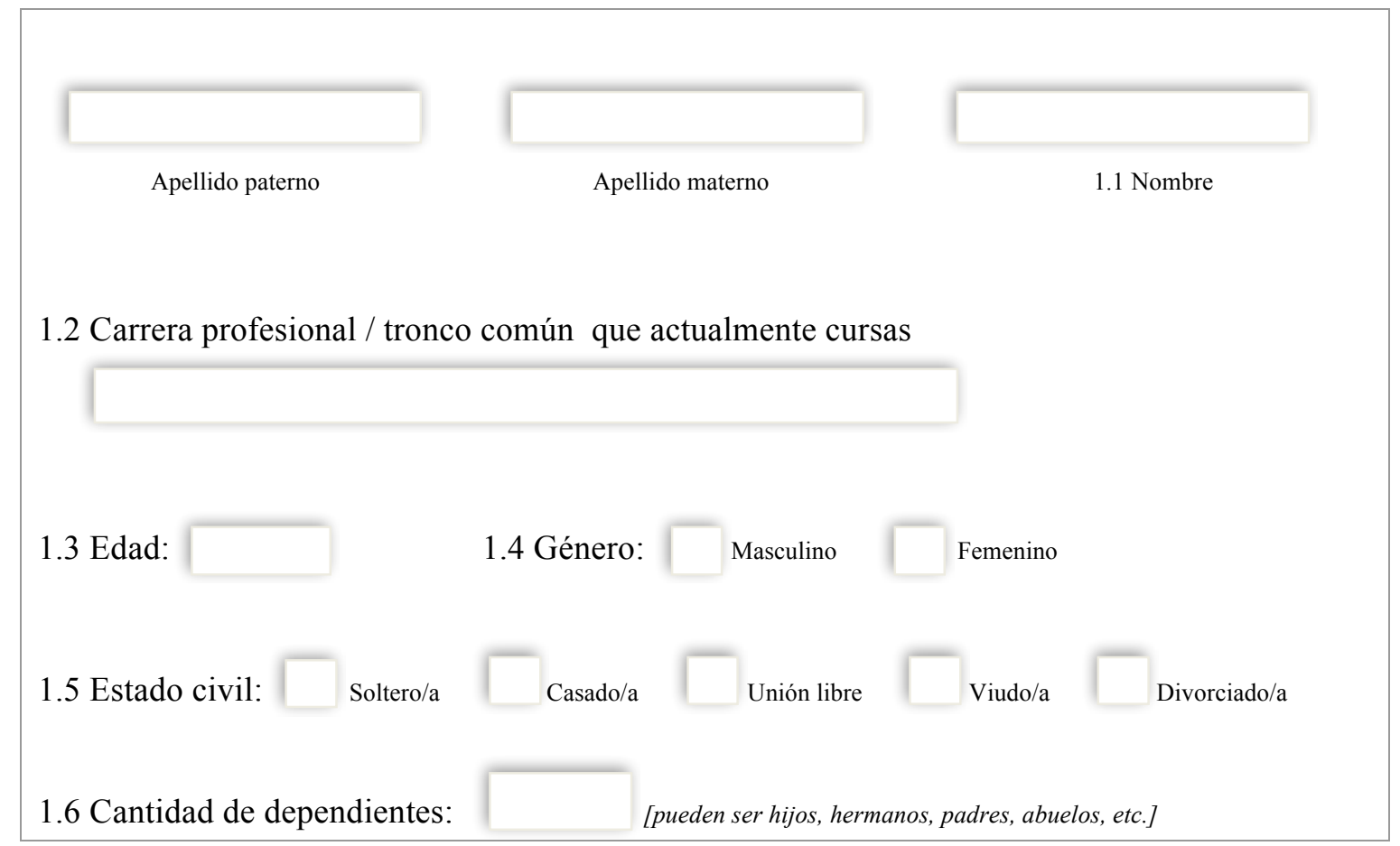




\section{TRAYECTORIA ESCOLAR Y CAPACITACIÓN TECNOLÓGICA}

2.1 Promedio de bachillerato:

2.2 Tipo de bachillerato de procedencia: Público Privado

2.3 Aproximadamente, ¿cuántos años de experiencia tienes usando la computadora?

2.4 Aproximadamente, ¿cuántos cursos en el área computacional has tomado?

2.5 Por favor, indica el grado de capacitación alcanzado con dicho(s) curso(s):

Introductorio (aspectos básicos)

Intermedio (manejo de ciertos programas: Word, Power Point, Excel, etc.)

Avanzado (funciones más complejas: programación, desarrollo multimedia,

etc.)

Ningún curso (autodidacta, en el caso de no haber realizado cursos)

\section{DATOS SOCIOECONÓMICOS Y DISPONIBILIDAD DE PC E INTERNET}

3.1 La casa donde actualmente vives es:

3.2 ¿Cuántas personas viven en tu casa?

$$
\text { No. de personas }
$$

3.3 Con relación a disponer de equipo de cómputo en en el hogar:

3.4 ¿Cuántas computadoras hay tu hogar?

$$
\begin{aligned}
& \text { No dispongo de PC } \\
& \text { Dispongo de PC } \\
& \text { Dispongo de PC + Internet }
\end{aligned}
$$

3.5 Los servicios de internet, principalmente los accedes en:
En casa

No accedo

3.6 Marca con una X la opción que más se aproxime a la escolaridad máxima alcanzada por tus padres: 


\begin{tabular}{|c|c|c|}
\hline 01. Ninguna & [ ] & [ ] \\
\hline 02. Estudios parciales de primaria & [ ] & [ ] \\
\hline 03. Primaria completa & {$[\quad]$} & [ ] \\
\hline 04. Estudios parciales de secundaria & {$[\quad]$} & {$\left[\begin{array}{l}1 \\
{[}\end{array}\right.$} \\
\hline 05. Secundaria & [ ] & [ ] \\
\hline 06. Carrera técnica & [ ] & {$[\quad]$} \\
\hline 07. Bachillerato o equivalente & {$[\quad]$} & [ ] \\
\hline 08. Normal/Normal superior & [ ] & {$\left[\begin{array}{ll}1 & \end{array}\right]$} \\
\hline 09. Estudios parciales de licenciatura & [ ] & [ ] \\
\hline 10. Pasante de licenciatura & [ ] & {$[\quad]$} \\
\hline 11. Licenciatura & [ ] & [ ] \\
\hline 12. Especialidad/Maestría & [ ] & {$[\quad]$} \\
\hline 13. Doctorado & [ ] & [ ] \\
\hline 14. Posdoctorado & [ ] & {$[\quad]$} \\
\hline 15. Lo ignoro & [ ] & [ ] \\
\hline
\end{tabular}

3.7 Marca con una X la opción que mejor represente la ocupación de tus padres:

\begin{tabular}{|c|c|c|}
\hline & Padre & Madre \\
\hline 01. Obrero(a) no especializado(a), trabajos en fábricas, maquiladoras, etc. & [ ] $]$ & [ ] \\
\hline 02. Obrero(a) especializado(a): electricidad, mecánica, cosméticos, etc. & {$[\quad]$} & {$[\quad]$} \\
\hline 03. Trabajo en oficinas, ventas (secretarial o administrativo) & {$[\quad]$} & {[} \\
\hline 04. Trabajo técnico (en laboratorios, en talleres, etc.) & {$[\quad]$} & [ ] \\
\hline 05. Trabajo docente (primaria, secundaria, técnica, bachillerato, etc.) & {$[\quad]$} & {$[\quad]$} \\
\hline 06. Trabajo docente nivel superior (Universidad, Tecnológico, Cetys) & {$[\quad]$} & {$[\quad]$} \\
\hline 07. Trabajo profesional (Ingeniería, Leyes, Medicina, etc.) & {$[\quad]$} & {$[\quad]$} \\
\hline 08. Responsable el hogar & {$[\quad]$} & {$[\quad]$} \\
\hline 09. Comerciante & {$[\quad]$} & {[} \\
\hline 10. Jubilado/Pensionado & {$[\quad]$} & [ ] \\
\hline 11. No trabaja & {$\left[\begin{array}{ll} & ]\end{array}\right.$} & {$\left[\begin{array}{ll} & ]\end{array}\right.$} \\
\hline 12. Finado & {$[\quad]$} & \\
\hline 13. Otra ocupación o empleo & {$[\quad]$} & {$[\quad]$} \\
\hline ¿Cuál? & & \\
\hline
\end{tabular}




\section{TIPO Y NIVEL DE USO TECNOLÓGICO}

En cada una de las siguientes preguntas, por favor marca con una X el recuadro que mejor refleje tu situación.

4.1 De manera personal, ¿qué tipo de computadora tienes?

Ninguna

PC de torre

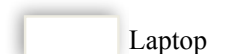

Tablet PC

Palm PC

Otro ¿Cuál?

4.2 Aproximadamente, ¿cuántas horas al día utilizas la computadora?

Menos de $1 \mathrm{hrs}$.

$1-2$ hrs.

3-4 hrs.

$5-6$ hrs.

Más de 7

4.3 Aproximadamente, ¿con qué frecuencia utilizas cada uno de los siguientes programas/medios computacionales?

\begin{tabular}{|c|c|c|c|c|c|c|}
\hline & & Nunca & $\begin{array}{c}\text { Algunas veces al } \\
\text { año }\end{array}$ & $\begin{array}{l}1-3 \text { veces } \\
\text { por mes }\end{array}$ & $\begin{array}{c}1-3 \text { veces } \\
\text { por } \\
\text { semana } \\
\end{array}$ & $\begin{array}{c}4 \text { ó más } \\
\text { veces por } \\
\text { semana }\end{array}$ \\
\hline a. & Procesador de texto (Word, por ejemplo) & {$[\quad]$} & [ ] & [ ] & {$[\quad]$} & [ ] \\
\hline b. & $\begin{array}{l}\text { Programas de presentación (Power Point, } \\
\text { por ejemplo) }\end{array}$ & [ ] ] & {$[\quad]$} & {$\left[\begin{array}{l}\text { [ } \\
{[}\end{array}\right.$} & [ $\quad]$ & [ ] \\
\hline c. & $\begin{array}{l}\text { Hojas de cálculo/bases de datos (Excel, por } \\
\text { ejemplo) }\end{array}$ & {$\left[\begin{array}{ll}\text { [ } \\
\text { ] }\end{array}\right.$} & {$\left[\begin{array}{ll}{[} & ]\end{array}\right.$} & {$\left[\begin{array}{ll}{[} & ]\end{array}\right.$} & {$\left[\begin{array}{ll}{[} & ]\end{array}\right.$} & {$\left[\begin{array}{ll}{[} & ]\end{array}\right.$} \\
\hline d. & $\begin{array}{l}\text { Paquetes matemáticos, estadísticos, de } \\
\text { diseño }\end{array}$ & {$\left[\begin{array}{ll}{[} & ]\end{array}\right.$} & {$\left[\begin{array}{ll}{[} & ]\end{array}\right.$} & {$\left[\begin{array}{ll}{[} & ]\end{array}\right.$} & {$\left[\begin{array}{ll}{[} & ]\end{array}\right.$} & [ [ ] \\
\hline e. & $\begin{array}{l}\text { Programa gráficos (Print Shop, Corel, por } \\
\text { ejemplo) }\end{array}$ & {$\left[\begin{array}{ll}{[} & ]\end{array}\right.$} & {$\left[\begin{array}{ll}{[} & ]\end{array}\right.$} & {$\left[\begin{array}{ll}{[} & ]\end{array}\right.$} & {$\left[\begin{array}{ll}{[} & ]\end{array}\right.$} & {$\left[\begin{array}{ll}{[} & ]\end{array}\right.$} \\
\hline f. & $\begin{array}{l}\text { Programa para el manejo multimedia } \\
\text { (audio, video etc.) }\end{array}$ & {$\left[\begin{array}{ll}{[} & ]\end{array}\right.$} & {$\left[\begin{array}{ll}{[} & ]\end{array}\right.$} & {$\left[\begin{array}{ll}{[} & ]\end{array}\right.$} & {$\left[\begin{array}{ll}{[} & ]\end{array}\right.$} & [ ] ] \\
\hline g. & $\begin{array}{l}\text { Enciclopedias u otras referencias en CD- } \\
\text { ROM }\end{array}$ & {$\left[\begin{array}{ll}\text { l } \\
\text { ] }\end{array}\right.$} & {$\left[\begin{array}{ll}{[} & ]\end{array}\right.$} & {$\left[\begin{array}{ll}{[} & ]\end{array}\right.$} & {$\left[\begin{array}{ll}{[} & ]\end{array}\right.$} & {$\left[\begin{array}{ll}{[} & ]\end{array}\right.$} \\
\hline h. & WWW & {$[\quad]$} & {$[\quad]$} & {$[\quad]$} & {$[\quad]$} & {$[\quad]$} \\
\hline i. & Correo electrónico (email) & {$[\quad]$} & [ ] & [ ] & [ ] & [ ] \\
\hline j. & Foros asincrónicos y chats & {$\left[\begin{array}{l}{[} \\
{[}\end{array}\right.$} & {$[\quad]$} & [ ] & {$[\quad]$} & {$[\quad]$} \\
\hline k. & Blogs & {$[\quad]$} & {$[\quad]$} & [ ] & [ ] & [ ] \\
\hline 1. & $\begin{array}{l}\text { Juegos/recreación (bajar películas, música, } \\
\text { imágenes, etc.) }\end{array}$ & {$[\quad]$} & {$[\quad]$} & {$[\quad]$} & [ ] & {$[\quad]$} \\
\hline
\end{tabular}

4.4 Considerando el listado anterior, escribe en cada recuadro la letra asociada al programa/medio según la importancia que tú le asignas para apoyarte en tus estudios.

$1^{\circ}$ más importante

$2^{\circ}$ más importante

$3^{\circ}$ más importante 
4.5 ¿Cómo estimas que es tu capacidad para usar cada uno de los siguientes programas/medios computacionales?

\begin{tabular}{|c|c|c|c|c|c|}
\hline & Pésima & Deficiente & Regular & Buena & Excelente \\
\hline Procesador de texto (Word, por ejemplo) & {$[\quad]$} & [ ] & [ ] & [ ] $]$ & [ ] \\
\hline Programas de presentación (Power Point, por ejemplo) & [ ] & [ ] & [ ] & {$[\quad]$} & [ ] \\
\hline Hojas de cálculo/bases de datos (Excel por ejemplo) & & [ ] & [ ] & {$[\quad]$} & {[} \\
\hline Paquetes matemáticos, estadísticos, de diseño & [ ] & [ ] & [ ] & [ ] & [ ] \\
\hline Programas gráficos (Print Shop, Corel, por ejemplo) & [ ] & [ ] & [ ] & {$[\quad]$} & {[} \\
\hline Programa para manejo multimedia (audio, video, etc.) & {$[\quad]$} & [ ] & [ ] & {$[\quad]$} & [ ] \\
\hline Enciclopedias u otras referencias en CD-ROM & [ ] & [ ] & [ ] & {$[\quad]$} & [ ] \\
\hline WWW & [ ] & [ ] & [ $\quad]$ & {$[\quad]$} & {$[\quad]$} \\
\hline Correo electrónico (email) & & [ ] & [ ] & {$[\quad]$} & {[} \\
\hline Foros asincrónicos y chats & [ ] & {$[\quad]$} & [ ] & {$[\quad]$} & [ ] \\
\hline Blogs & [ ] & [ ] & [ ] & {[} & {[} \\
\hline Juegos/recreación (bajar películas, música, imágenes, etc.) & [ ] & [ ] & [ ] & {$[\quad]$} & [ ] \\
\hline
\end{tabular}

4.6 ¿Qué tan eficiente te consideras para realizar cada una de las siguientes actividades con la computadora?

\begin{tabular}{|c|c|c|c|c|c|}
\hline & Pésimo & Deficiente & Regular & Bueno & Excelente \\
\hline Organización de información (archivos) en carpetas & [ ] & [ ] & [ ] & {$[\quad]$} & [ ] \\
\hline Transferencia de archivos (entre carpetas, CD, USB, etc.) & [ ] & [ ] & [ ] & [ ] & [ ] \\
\hline $\begin{array}{l}\text { Elaboración de documentos con formato adecuado e inserción } \\
\text { de tablas y gráficos, entre otros }\end{array}$ & [ ] & {$[\quad]$} & {$[\quad]$} & {$[\quad]$} & {$[\quad]$} \\
\hline Elaboración avanzada de hojas de cálculo & [ ] & {$[\quad]$} & {$[\quad]$} & {$[\quad]$} & {$[\quad]$} \\
\hline Diseño y desarrollo de bases de datos (MySQL, por ejemplo) & [ ] & [ ] & {$[\quad]$} & {$[\quad]$} & [ ] \\
\hline Creación de presentaciones & {$[\quad]$} & {$[\quad]$} & {$[\quad]$} & {$[\quad]$} & {$[\quad]$} \\
\hline Manejo de imágenes/fotos & {$[\quad]$} & {$[\quad]$} & {$[\quad]$} & {$[\quad]$} & {$[\quad]$} \\
\hline $\begin{array}{l}\text { Procesamiento estadístico de datos (manejo de SPSS, } \\
\text { Statistica u otros programas, por ejemplo) }\end{array}$ & [ ] & [ ] & [ ] & [ ] $]$ & [ ] \\
\hline Búsqueda de información vía Internet & {$[\quad]$} & {$[\quad]$} & {$[\quad]$} & {$[\quad]$} & {$[\quad]$} \\
\hline $\begin{array}{l}\text { Establecimiento de comunicación vía electrónica (email, foros, } \\
\text { chats, blogs, etc.) }\end{array}$ & {$[\quad]$} & [ ] & [ ] & {$[\quad]$} & \\
\hline Creación de documentos multimedia (audio, imágenes, video) & [ ] & [ ] $]$ & [ ] & [ ] $]$ & [ ] \\
\hline
\end{tabular}


4.7 De las siguientes actividades derivadas del uso de la computadora, por favor escribe en los recuadros ubicados a la derecha la letra asociada según la importancia y el uso que le asignas.

a. Elaborar proyectos, ensayos, tareas, etc.

b. Crear presentaciones

c. Trabajar con bases de datos/hojas de cálculo

d. Elaborar documentos multimedia

e. Practicar habilidades (escritura, lectura, operaciones matemáticas, etc.)

$1^{\circ}$ más importante

$2^{\circ}$ más importante

$3^{\circ}$ más importante

f. Buscar información (Internet, enciclopedias en CD-ROM, etc.)

g. Comunicarse por correo electrónico (email)

h. Intercambiar opiniones en foros asincrónicos y chats

i. Crear y mantener blogs

j. Manejar información/crear sitios web

k. Jugar/recrearse (bajar películas, música, imágenes, etc.)

I. Otro (¿Cuál?)

4.8 ¿En cuál nivel te ubicarías de acuerdo a tu relación con la tecnología computacional? Marca sólo uno.

\begin{tabular}{|c|c|c|}
\hline Nivel & Descripción & \\
\hline Conciencia & $\begin{array}{l}\text { Estoy conciente de que existe la tecnología pero no la he usado, quizás hasta trato de } \\
\text { evitarla. Me causa ansiedad la sola idea de usar una computadora }\end{array}$ & [ ] \\
\hline $\begin{array}{l}\text { Aprendiendo el } \\
\text { proceso }\end{array}$ & $\begin{array}{l}\text { Actualmente estoy tratando de aprender las bases. Algunas veces me siento frustrado } \\
\text { usando computadoras. No siento confianza }\end{array}$ & [ ] \\
\hline $\begin{array}{l}\text { Entendimiento y } \\
\text { aplicación }\end{array}$ & $\begin{array}{l}\text { Estoy comenzando a entender el proceso de usar la tecnología y puedo pensar en tareas } \\
\text { específicas en donde me podría ser útil }\end{array}$ & [ ] \\
\hline $\begin{array}{l}\text { Familiaridad y } \\
\text { confianza }\end{array}$ & $\begin{array}{l}\text { Estoy ganando confianza al usar la computadora para tareas específicas. Comienzo a } \\
\text { sentirme a gusto cuando la uso }\end{array}$ & [ ] \\
\hline $\begin{array}{l}\text { Adaptación a otros } \\
\text { contextos }\end{array}$ & $\begin{array}{l}\text { Pienso en la computadora como una herramienta útil y ya no me atemoriza que sea } \\
\text { tecnología. Puedo usarla en muchas aplicaciones }\end{array}$ & [ ] \\
\hline $\begin{array}{l}\text { Aplicación creativa } \\
\text { a contextos nuevos }\end{array}$ & $\begin{array}{l}\text { Puedo aplicar lo que conozco de tecnología en mis labores académicas y extra-académicas. } \\
\text { Soy capaz de usarla para muchas tareas, en diferentes formas y para muchos objetivos }\end{array}$ & {$\left[\begin{array}{ll}\text { [ } \\
\text { ] }\end{array}\right.$} \\
\hline
\end{tabular}


4.9 ¿En cuál nivel de uso del web te ubicarías? Marca sólo una opción.

\begin{tabular}{|c|c|c|}
\hline Nivel & Descripción & \\
\hline Informativo & Sólo consulta de información general, por ejemplo, noticias, avisos, etc. & [ ] \\
\hline Suplementario & $\begin{array}{l}\text { Obtención de información complementaria para tareas, por ejemplo, archivos Power Point, } \\
\text { documentos pdf, etc. }\end{array}$ & {$[\quad]$} \\
\hline Esencial & Requiero acceder al web para ser productivo en mis estudios & [ ] \\
\hline Comunitario & Soy un usuario frecuente de Internet y colaboro con archivos para mantener un sitio web & {$[\quad]$} \\
\hline Inmersivo & Dependo del web en la búsqueda de información, procesamiento, comunicación, etc. & [ ] \\
\hline
\end{tabular}

\section{OPINIÓN ACERCA DEL USO DE LA TECNOLOGÍA}

5.1 Marca con una $\mathrm{X}$ dentro del recuadro que mejor refleje tu opinión acerca del rol de la tecnología computacional en los procesos de aprendizaje.

La tecnología computacional puede fortalecer el proceso de aprendizaje

El aprendizaje es independiente del uso de la tecnología computacional

5.2 Completa los recuadros junto a cada frase con una de las siguientes opciones

\section{$\boldsymbol{0}=$ Totalmente en desacuerdo; $\boldsymbol{1}=$ En desacuerdo; $\mathbf{2}=$ Indeciso; $3=$ De acuerdo; $\mathbf{4}=$ Totalmente de acuerdo}

La computadora es muy importante en mis estudios tareas

El uso de la computadora mejora mi forma de

expresión escrita

El uso de la computadora en clases me motiva motiva como estudiante

La computadora fortalece mi capacidad academico de investigación

Creo que es muy importante para mí aprender a usar la computadora

El uso de la computadora me ayuda a hacer mejores

El uso de la computadora es trascendente en los procesos

educativos

El uso de la computadora será importante en mi futura profesión

El uso de la computadora mejora mi desempeño

El uso de la computadora me ayuda a profundizar los conceptos vistos en clases

La computadora propicia el aprendizaje de temas

El uso de la computadora permite extender el proceso de aprendizaje más allá de las clases 